\title{
STUDY OF FOUR-WHEEL STEERING SYSTEMS' VIABILITY IN FUTURE AUTOMOBILES
}

\author{
Aayush Soni \\ Department of Mechanical Engineering \\ Bhilai Institute of Technology \\ Durg, Chhattisgarh, India \\ Prakhar Shrivastava \\ Department of Mechanical Engineering \\ Bhilai Institute of Technology \\ Durg, Chhattisgarh, India
}

\begin{abstract}
Presently, all vehicles have a two-wheel steering system irrespective of the vehicle being front wheel driven, rear wheel driven or all-wheel driven. A four-wheel steering system known as "quadra steering" system is a system in which both the front wheels and rear wheels get steered according to the speed of the vehicle and space available for turning. This system makes the vehicle more stable and enhances its performance. In this report, the performance of quadra steering system has been considered under low speed, medium speed and high-speed conditions. For parking and low speed conditions, rear wheels are turned in the opposite directions while at medium and high-speed conditions, rear wheels and front wheels are turned in the same direction. As a result, the vehicle becomes more stable and its turning radius reduces greatly.
\end{abstract}

Keywords - Four Wheel Steering System, Steering System, Quadra Steering System, Steering Mechanism

\section{INTRODUCTION}

The function of a steering system is to convert the rotary movement of steering wheel in driver's hand into angular turn of the front wheels on road. The steering system is designed to enable the driver to control and continuously adjust the steered path of

\author{
Adarsh Sahu \\ Department of Mechanical Engineering \\ Bhilai Institute of Technology \\ Durg, Chhattisgarh, India \\ Dr. (Mrs.) Shubhrata Nagpal \\ Department of Mechanical Engineering \\ Bhilai Institute of Technology \\ Durg, Chhattisgarh, India
}

the vehicle. The perfect steering is achieved when all the four wheels are rolling perfectly under all condition of running, while taking turns the condition of perfect rolling is satisfied if the axes of front wheels when produced meet the rear wheel axis at one point. This point is called as the instantaneous centre of vehicle.

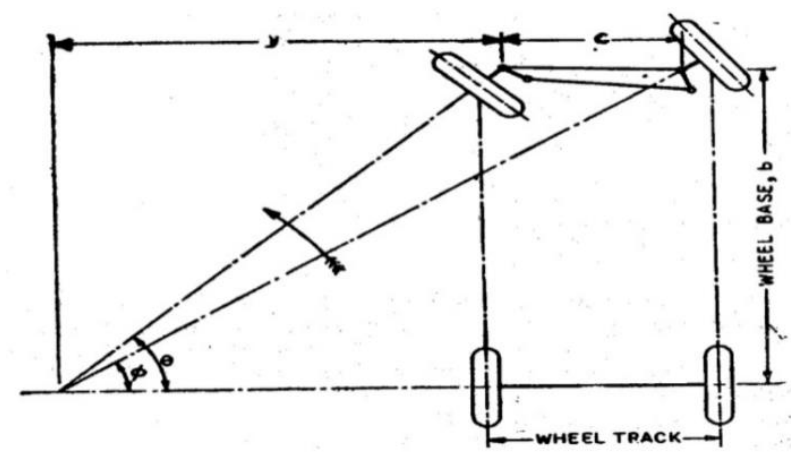

Fig. 1: Steering of a four wheeled vehicle

It is seen that the inside wheel is required to turn through a greater angle than the outer wheel. The larger is the steering angle, the smaller is the turning radius. The smallest circle which front wheels can traverse, which is obtained when two wheels are at extreme position is known as turning circle.

For correct steering, 


$$
\begin{gathered}
\cot \varnothing=\frac{y+c}{b}=\frac{y}{b}+\frac{c}{b} \\
\cot \varnothing=\cot \theta+\frac{c}{b} \\
\cot \varnothing-\cot \theta=\frac{c}{b}
\end{gathered}
$$

Where,

$\mathrm{c}=$ lateral wheel separation

$\mathrm{b}=$ longitudinal wheel separation

$\theta=$ Relative angle of inside wheel

$\emptyset=$ Relative angle of outside wheel

\section{LITERATURE REVIEW}

1. K.Lohith [1] has designed a four wheel steering system taking Maruti Suzuki 800 as the benchmark vehicle. [1] has also given introduction of four-wheel Steering System, advantages of four-wheel steering over conventional two-wheel steering system and application of four-wheel steering in parallel parking and high-speed lane changing.[4] has designed the steering system model in CATIA and ADAMS software and has made calculations for steering angle, radius and instantaneous centre. A comparison graph is also drawn between Steering Wheel and rear and front wheels. Turning radius has also been compared of two wheel and four-wheel steering system.

2. Dilip S Choudhari [2] has comprehensively analysed the four-wheel steering system, detailing its working as well as the phases of its working i.e., positive, negative and neutral. The paper also describes the difference between fourwheel and the conventional two-wheel steering system. The paper however didn't provide many practical results. Also, no actual sized vehicles were used to produce the provided practical results.

3. Arun Singh [3] has provided a good detailed description of a four-wheel steering system in automobiles. The paper includes thorough description of the technical details, modes as well as the components of working of such a steering system. The paper also outlines the advantages and disadvantages of a four-wheel steering system. However, sufficient data is not available on the cost effectivity of such a system. Moreover, almost no practical results are available comparing the four-wheel and twowheel steering systems.

4. Amandeep [4] described the types of steering mechanism used in steering systems of fourwheeled automobiles. [5] has also discussed about the gears used, components required for steering system design and various modes in which four-wheel steering system operates. Comparison between four wheel and two-wheel steering systems have also been made. The paper however does not provide sufficient details regarding the cost of design and failures in steering system.

5. Ahmed [5] has described the different mechanisms used in steering system and an improved and better Ackerman system, with the help of which, the rear wheels can also be turned with respect to the direction of front wheel. The paper also provides the practical application of this system; however, it does not provide the designing techniques of this system.

\section{TYPES OF STEERING MECHANISMS}

\section{$\underline{\text { Ackerman steering mechanism }}$}

Ackerman steering mechanism is almost used universally because of its simplicity. The Ackerman steering mechanism is a geometric arrangement of linkage in the steering of a vehicle designed to turn the inner and outer wheels at appropriate angles.

It consists of cross-link $\mathrm{CD}$ connected to the short axle $\mathrm{AL}$ and $\mathrm{BM}$ of the two front wheels through the short arms $\mathrm{AC}$ and $\mathrm{BD}$, thus forming a bell crank lever LAC and MBD.

So, when the wheel is running straight, $C D$ is parallel to $\mathrm{AB}$ and the short arms $\mathrm{AC}$ and $\mathrm{BD}$ both makes equal angle $\alpha$ to the horizontal axis of chassis. 


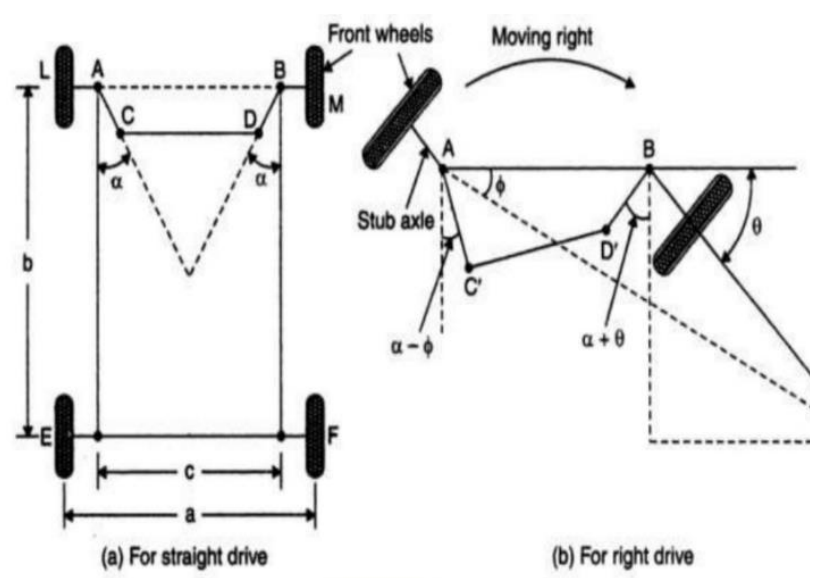

Fig. 2: Ackerman steering mechanism

In order to satisfy the fundamental equation of corrected steering, links AC and BD are suitably proportioned and angle $\alpha$ is suitably selected.

For correct steering,

$$
\cot \varnothing-\cot \theta=\frac{c}{b}
$$

Value of $\frac{c}{b}$ lies between 0.4 to 0.5

Relation between $\alpha, \beta$ and $\emptyset$ is given by-

$$
\tan \alpha=\frac{\sin \emptyset-\sin \theta}{\cos \emptyset+\cos \theta+2}
$$

\section{Davis steering mechanism}

Davis steering mechanism has sliding pairs instead of turning pairs, thus there is more friction acting and easy wear and tear of components. Ultimately, it becomes inaccurate after a certain time.

It consists of cross-link $\mathrm{CD}$ sliding parallel to another link $\mathrm{AB}$ and is connected to stub axle of the two front wheels by means of two similar bell crank levers LAC and MBD pivoted at $\mathrm{A}$ and $\mathrm{B}$ respectively. The link CD slides in the bearing and carries sliding pair at its ends $\mathrm{C}$ and $\mathrm{D}$.

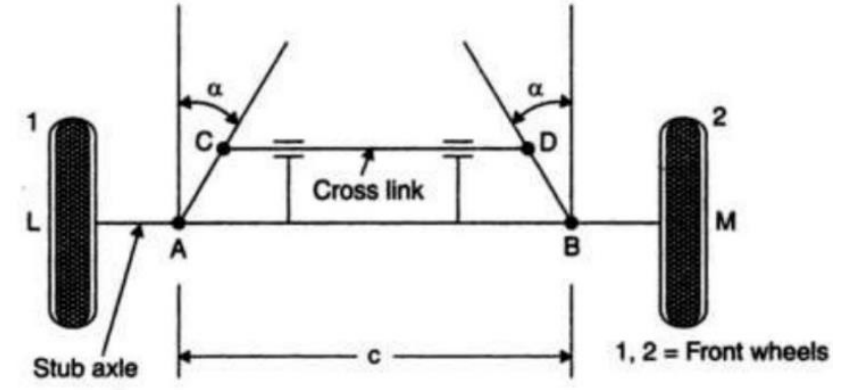

Davis steering gear mechanism.

Fig. 3: Davis steering mechanism

When the wheel is running straight, the gear is said to be in its mid position. The correct steering depends upon the suitable selection of cross arm angle $\alpha$ and is given by-

$\tan \alpha=\frac{c}{2 b}$

where, $\mathrm{c}$ is the distance between pivots of front axle and $b$ is wheelbase.

Here, $\frac{c}{2 b}$ lies between 0.4 to 0.5 and

$\alpha$ lies between $11.3^{\circ}$ and $14.1^{\circ}$.

The Davis steering mechanism is theoretically correct but due to presence of more sliding members the wear is increased, thus becoming inaccurate. Hence, this system is not used commonly.

\section{STEERING MECHANISM}

Steering allows the driver to guide or turn the moving vehicle in desired direction without the slipping of wheels. In conventional steering system, only the front wheels are turned left or right as desired while rear wheels stay straight. Just by tilting the front wheels, the whole vehicle can be turned.

\section{PHYSICS OF WHEELS}

In a wheel which is rolling perfectly, the velocity of wheel at contact point must be zero. This is possible because a wheel has two types of motion, Linear and Rotational. 


\section{ROLLING WITHOUT SLIPPING}

Velocity at any point on a rolling object

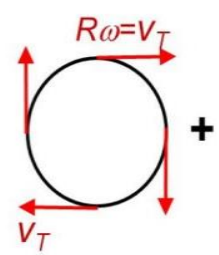

Velocity at point

in pure ROTATION (in CM ref frame)

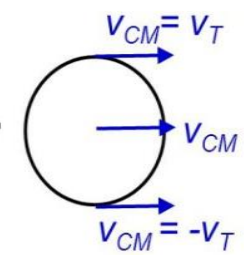

$\begin{gathered}\text { Velocity at point } \\ \text { in }\end{gathered}$
pure TRANSLATION
(relative to ground)

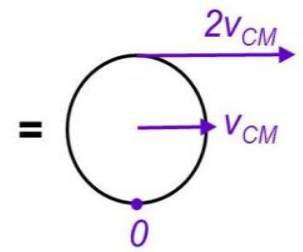

Velocity at point in COMBINED ROLLING MOTION
Fig.4: Rolling without slipping

Linear and rotational velocity at bottom cancel each other. Hence, velocity at contact point $\mathrm{V}=0$

Here, $\mathrm{V}_{\mathrm{CM}}=$ Velocity of Center of Mass

$$
\mathrm{V}_{\mathrm{T}}=\text { Tangential Velocity }
$$

Assuming that even after the wheel is turned, the vehicle continues on a straight path. In this case, the rotational velocity and translational velocity will not cancel each other and the vehicle will skid. The only way to achieve zero velocity at point of contact is to make sure that translation velocity is also inclined. Therefore, the vehicle should turn.

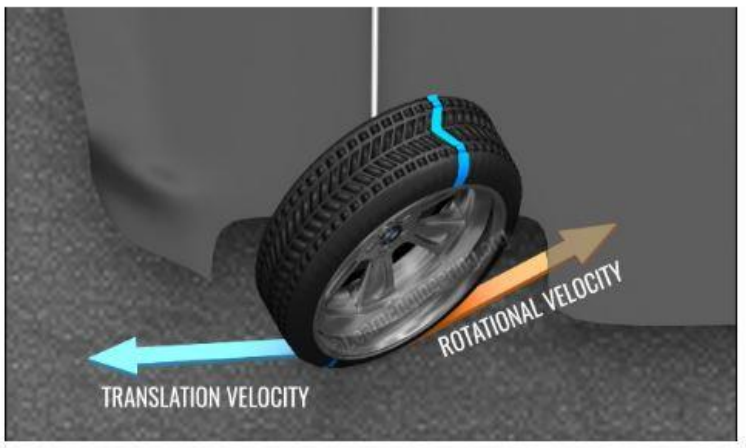

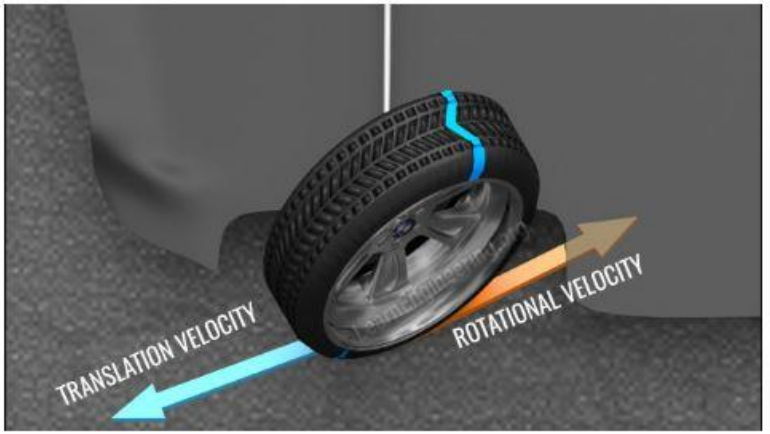

Fig.5: Translational \& Rotational velocity of wheels

\section{STEERING MECHANISM}

The most commonly used steering mechanism in modern vehicle is Rack and Pinion. Rack and pinion system can convert rotational motion to translational motion.

\section{Rack-and-pinion gear}

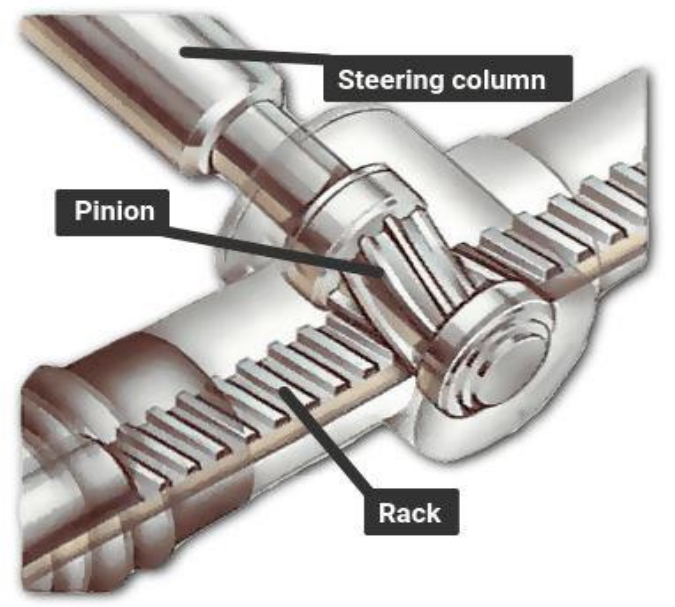

Fig.6: Rack \& Pinion arrangement

"Pinion" is a very small driving gear while "Rack" can be thought of as a gear of infinite Pitch Circle radius. Rack is constrained to move translationally.

This is manual steering which requires the driver to put a lot of effort to turn the wheels but now-a-days, electric power steering is used to rotate the pinion. Electronic control unit (ECU) measures various parameters like torque applied by the driver, steering angle, vehicle speed, steering wheel speed and then gives appropriate power to the motor. The power of 


\section{International Journal of Engineering Applied Sciences and Technology, 2021 \\ Vol. 6, Issue 2, ISSN No. 2455-2143, Pages 258-266 \\ Published Online June 2021 in IJEAST (http://www.ijeast.com)}

motor is given to the steering shaft via a worm gear and planetary gear set.

\section{FOUR WHEEL STEERING SYSTEM}

It is also known as "Quadra steering system". In this system, both front and rear wheels can be tilted to make a turn in small space.

\section{Phases of four-wheel steering}

\section{- $\quad$ Negative Phase}

In this phase, rear and front wheels move in opposite directions. It is used when vehicle speed is low. While using this system, the vehicle can be turned in a very small space. So, it is used while parking.

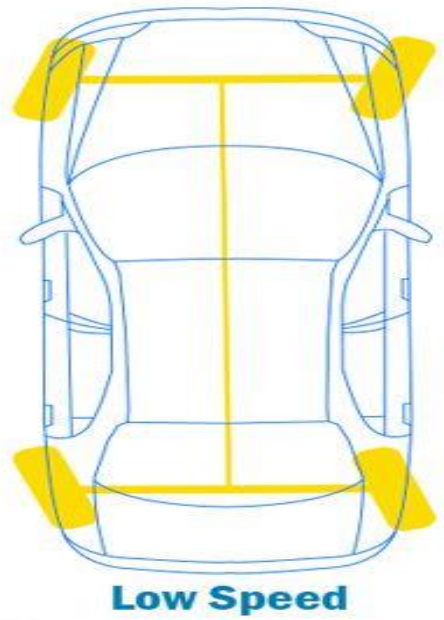

Fig.7: Negative Phase Steering

\section{- Positive Phase}

In this phase, rear and front wheels are moved in the same direction. The vehicle Moves Like a crab rather than in a curved path. It is used when the vehicle speed is high. It helps in changing lanes on highways at high speed. It decreases the centrifugal effect and cornering force while turning and increases the stability of the vehicle.

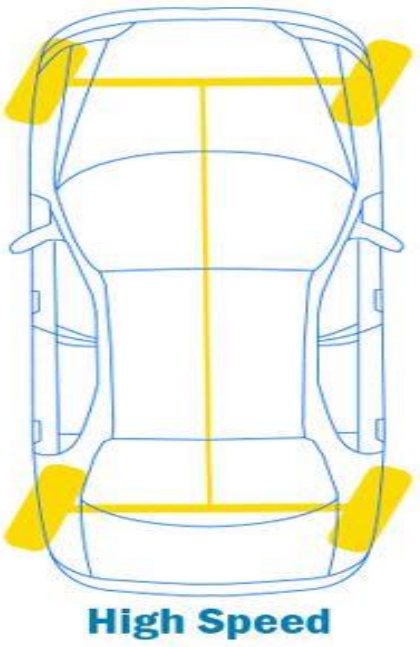

Fig.8: Positive Phase Steering

\section{- Neutral Phase}

In this phase, only the front wheels are turned while the rear wheels remain straight. This system is the normal front wheel steering which is in use currently in almost all vehicles.

\section{SOME BASICS RELATED TO DIFFERENTIAL AND WHEEL ALIGNMENT}

Differential and wheel alignment have a significant role in stability and handling of vehicle.

\section{Differential}

We can see from the figure that while taking a turn inner wheels have to cover a smaller distance than the outer wheels, which means that speed of inner wheels should be lesser than outer wheels. This is where differential comes into play. Differential is a mechanism which allows the wheels to rotate at different speeds

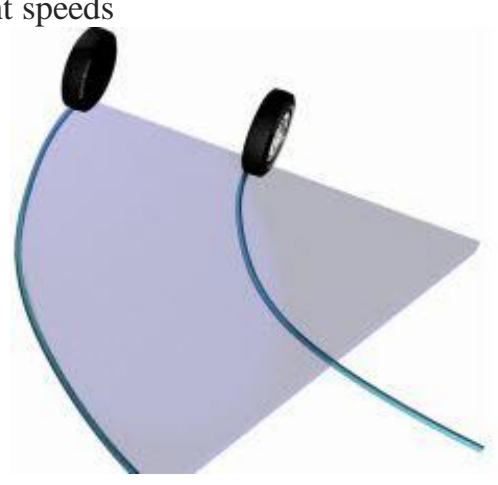

Fig.9: Turning radius of inner and outer wheels 


\section{2. $\underline{\text { Camber }}$}

When viewed from the front we can see that wheels are inclined at an angle to an axis perpendicular to the road. This angle is called "camber angle". It can also be defined as inwards or outwards tilt of tires when viewed from the front. It helps in distributing the load across the entire thread and improves handling.

Improper camber can make the tire wear on one edge and may cause the vehicle to pull to the side which has the most positive camber. Zero camber will give most uniform tire wear but it may decrease performance of vehicle during cornering.

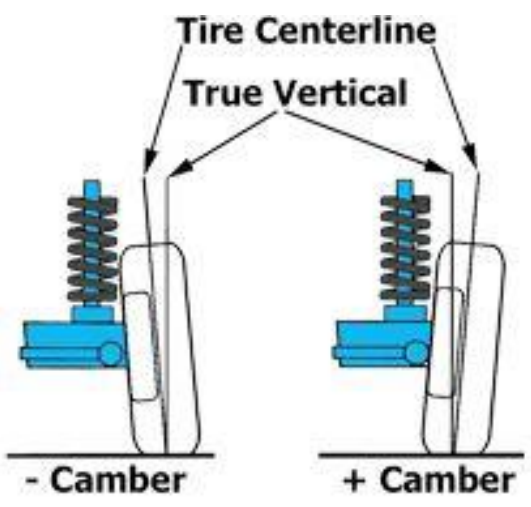

Fig.10: Camber

\section{Caster}

When viewed from the sides the angle between perpendicular to road and steering axis is called "caster angle". The steering axis is not vertical but slightly tilted. It gives good stability to the vehicle, helps to maintain straight direction and helps in selfcentering of the steering wheels.

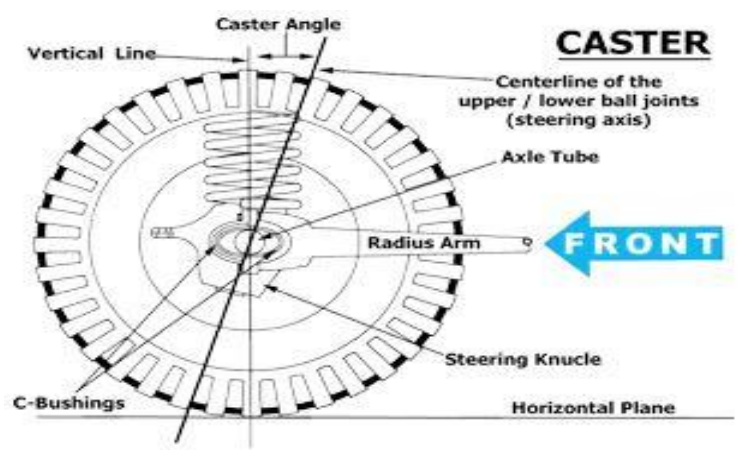

Fig. 11: Caster

\section{Toe In and Toe Out}

When viewed from above, Toe is the side-to-side difference in distance between front and rear side of front tires. If distance between the front side of tires is greater than rear side, it is called Toe-In and when vice-versa, it is called Toe-out.

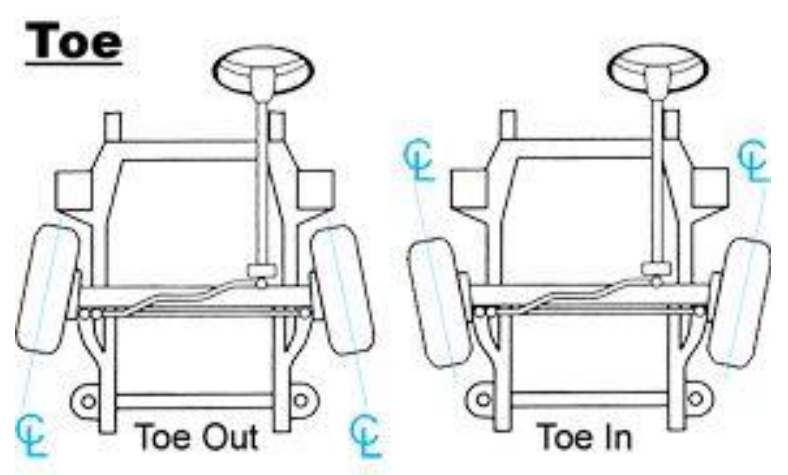

Fig.12 Toe-In \& Toe-Out

\section{COMPONENTS}

\section{Primary components}

- Vehicle speed sensors: These sensors interpret the speed of the vehicle's wheel rotation and send a signal to the electronic control unit. Two sensors, one within the speedometer and the other at the transmission output are used so that the most accurate results are obtained.

- Steering phase control unit: This unit sends signal to the power steering cylinder booster valve conveying the direction and stroke of rear wheel steering by the 
combined movement of the control yoke angle and bevel gear revolutions

- Electric stepper motor: It alters the yoke angle and bevel gear phasing.

- Rear steering shaft: It transmits the front wheel steering angle by turning the small bevel gear in the steering placement control unit which consequently causes the main bevel gear in the assembly to rotate.

- Control valve: It feeds hydraulic pressure to the steering actuator according to the phase and stroke required for optimal rear wheel steering.

- Hydraulic power cylinder: It runs the output rod by hydraulic pressure thus, steering the rear wheels. It locks the rear wheels in the neutral (straightforward) position with the centering lock spring in case of failure. his is activated by using a solenoid valve and ensures a normal twowheel steering system.

- Hydraulic pump: It provides hydraulic pressure to the front and rear steering systems.

\section{Steering phase control unit}

This unit changes the direction and degree to which the rear assembly gets steered. It has a stepper motor controlling the rear steering ratio, a control yoke, a swing arm, a main bevel gear attached to the rear steering shaft through a small bevel gear and a control rod connected to the control valve. It maintains that:

- Opposite phase steering is under 35 kilometers per hour.

- Control yoke is that is at an angle optimised by the stepper motor.

- Front wheels are steered to the right. The small bevel gear is rotated in " $\mathrm{X}$ " direction due to the rotation of real steering shaft. In turn, the small bevel gear rotates the main bevel gear.

- Rotation of the main bevel gear causes the control rod to move towards the control valve.

- Input rod of the control valve is pushed to the right, according to the degree of the control rod's movement, which is positioned to move in an upward direction, to the right. Thus, the rear wheels are steered to the left, opposite to the front wheels.

- As the angle of the control yoke is increased in direction A as vehicle speed decreases, the rear-to-front steering ratio increases accordingly, hence tightening the vehicle's steering lock.

- Neutral phase at 35 kilometers per hour, the control yoke's angle is horizontal (neutral). Therefore, the input is unaffected even if the control rod moves with the rotation of the bevel gear unit. Consequently, the real wheels are not steered in this mode.

\section{WORKING MECHANISM}

- It consists of a rack-and-pinion front steering system. This system is hydraulically powered by a twin tandem pump.

- The rear wheel steering mechanism is also hydraulically powered by the main pump and electronically controlled optimally according to the front steering angle and vehicle speed.

- The rear steering shaft extends from the rack bar of the front steering gear assembly to the rear steering phase control unit.

- The rear steering system comprises of the input end of the rear steering shaft, vehicle speed sensors, a steering phase control unit, a power cylinder and an output rod.

- A centering lock spring is added which locks the rear system in a neutral (straightforward) position in case the hydraulic system fails. Furthermore, a solenoid valve that disengages hydraulic assist and consequently activates the centering lock spring in case of an electric failure is also added.

- The 4WS alters the phase and ratio of the rear wheel steering in accordance with the vehicle speed.

- It steers the rear wheels in the opposite direction of the front wheels when speed is less than 35 kilometers per hour for a tighter turn and keeps them in a straightforward position when turning at 35 kilometers per hour. 
- Above 35 kilometers per hour, the system steers the rear wheels in the same direction as that of the front wheels, hence generating an increase cornering force for stability.

- The maximum steering angle of the rear wheels extends up to 10 degrees.

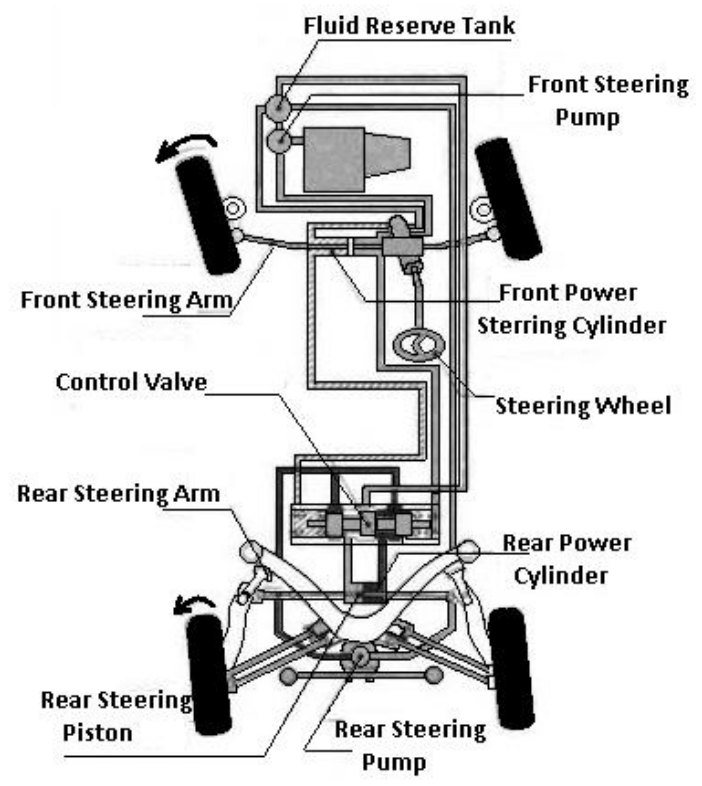

Four Wheel Steering System

Fig.13: Four-wheel steering system

\section{ADVANTAGES}

- Superior cornering stability: The vehicle displays more stable cornering behavior and controllability at high speed and on wet, slippery surfaces.

- Improved steering response and precision: The vehicle displays a quicker and more precise response to steering throughout its speed range.

- High-speed straight-line stability: The vehicle's ability to run in a straight line at high speed is announced. Negative effects of road irregularities and crosswinds are minimized.

- Improved rapid lane-changing stability: The vehicle's stability when changing lanes at high speed is improved. The vehicle is less likely to go into a spin even during distress situations.

- Smaller turning radius: When the rear wheels are steered in an opposite direction to the front wheels at low speed, the vehicle's turning radius becomes much smaller. This makes for easier maneuvering of the vehicle on narrow roads and during parking.

- Controlling: Computer controlled quadra steering system can be turned on and off and has an effective trailer towing mode.

\section{DISADVANTAGES}

- Expensive: The four-wheel steering system due to construction of many new components becomes expensive.

- Possibility of being inoperative: Since the system has a large number of electronic components, there is always a possibility of the non-functioning of one part rendering the complete system inoperative.

\section{APPLICATIONS}

- Parking: By counter direction steering of the rear wheels, a much smaller turning radius is possible in the $4 \mathrm{WS}$. Therefore, the vehicle turns in a small radius when parking.

- Junctions: On crossroads or junctions where roads intersect at 90 degrees or tighter angles, the counter direction steering of the rear wheels makes the turn easier.

- Slippery road surfaces: On low friction surfaces, the steering of rear wheels nullifies the sideways drift of the vehicle's rear end.

- High-speed straight-line operation: When a vehicle travels in a straight line at high speed, the driver has to make frequent steering corrections to maintain the required direction. (Lohith, 2013)Steering of the rear wheels in the same direction minimizes the need for these corrections.

- Narrow roads: On narrow roads with tight bends, counter direction steering of the rear wheels makes the vehicle's turning radius smaller and hence reduces the side-to-side 
rotation of the steering wheels making the vehicle easier to run.

- U-Turns: U-turns can be performed easily on narrow roads as the turning radius becomes smaller.

\section{CONCLUSION}

In the current state of research, no method has been found that can be used to design a four-wheel steering system in a cost-effective manner. Less research work has been reported on the practicality of such a system, other than in high-end luxury and sports cars. Future developments should focus on making the system more cost effective so that it can be installed in more general vehicles. With its growing popularity, it can be expected that it will become cheaper and come in more and more new vehicles in the future.

\section{REFERENCES}

1. Lohith, K. (2013). Development of Four Wheel Steering System for a Car. SASTECH Journal

2. Choudhari, D. S. (2014). Four Wheel Steering System for Future. International Journal of Mechanical Engineering and Robotics Research.

3. Singh, A. (2014). Study of 4 Wheel Steering Systems to Reduce Turning Radius and
Increase Stability. International Conference for Advanced Research and Innovation.

4. Amandeep. (2017). Four Wheel Steering System For Automobiles. International Journal for Research in Applied Sciences and Engineering Technology.

5. Ahmed, S. S. (2018). Four-Wheel Three Mode Steering System. International Journal of Academic Research and Development.

\section{WEBSITES}

1) https://www.theengineerspost.com/quadrasteering-system/

2) https://www.slideshare.net/shaikusmansha $\mathrm{g} / 7$-steering-mechanisms

3) https://www.howacarworks.com/basics/ho w-the-steering-system-works

4) https://physics.stackexchange.com/questio ns/308491/angular-velocity-of-a-wheel

5) https://learnengineering.org/understanding -anti-lock-braking-system.html

6) https://oards.com/four-wheel-steeringsystem-info/

7) https://explorationoutfitters.com/pages/cas ter-camber-toe-explained

8) https://learnengineering.org/how-does-adifferential-work.html 